Bajopas Volume 2 Number 1 June, 2009

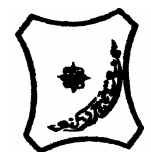

Bayero Journal of Pure and Applied Sciences, 2(1):160 - 167

Received: February, 2009

Accepted: May, 2009

\title{
A SIMULATION OF 450MHz AMPLIFIER WITH DISTRIBUTED OUTPUT USING BIPOLAR JUNCTION TRANSISTOR FROM NI- CIRCUIT DESIGN
}

\author{
*Gidado, A.S. and Ali, M.H. \\ Department of Physics, Bayero University, P.M.B. 3011, KANO \\ *Correspondence Author: abshugida@hotmail.com
}

\begin{abstract}
A study of the frequency response of a single stage common emitter amplifier, emitter -coupled amplifier and multistage distributed amplifier is carried out. In this work, a single stage common emitter amplifier is designed. Two such amplifiers were connected in a differential pair and designed. In this second design, the coupling between the stages is provided by the emitter resistor which carries the combined currents of the pair. From the previous stages, the multistage distributed amplifier was also designed. Such an arrangement employs two transmission lines, one for the input and the other for the output. Results obtained from simulation exercise indicate significant improvement in the gain, bandwidth and gain bandwidth product of the distributed amplifier.
\end{abstract}

Keywords: Simulation, Amplifier, Bipola, Transmitter, Circuit Design

\section{INTRODUCTION}

The increasing volume of data transported in the backplane of the internet, optical communication at rates of $40 \mathrm{~Gb} / \mathrm{s}$ has become attractive. Such high speeds emerge as a new territory for IC design because prior work of these frequencies ('millimetre - wave frequencies' ) has been limited to narrowband, low complexity circuits for wireless applications (Behzad, 2003 ). The need for demanding higher gain, bandwidth and gain bandwidth product of any electronic systems arises due to the important attaches to the operation of these devices at higher frequencies. Thus, three critical parameters, namely, bandwidth, signal power and noise are the most important parameters in determining the performance of any given communication system (Bencman and Hajimiri, 2004). The inherent capacitors of such devices are the main causes of bandwidth limitation in wideband amplifiers. Several bandwidth enhancement methods have been proposed in the past. A more exotic approach to solving the problem was proposed by Ginzton etal. using distributed amplification(Charles and Emilio, 2003).

Single Stage Common Emitter Amplifier

The basic circuit for Hybrid pi model for low and high frequencies analysis are shown below. The biasing capacitors and resistors necessary to keep the active region are also shown(http://www.mems.ee.metu.edu.tr/courses/ee313/project2007.pdf24/03/08)

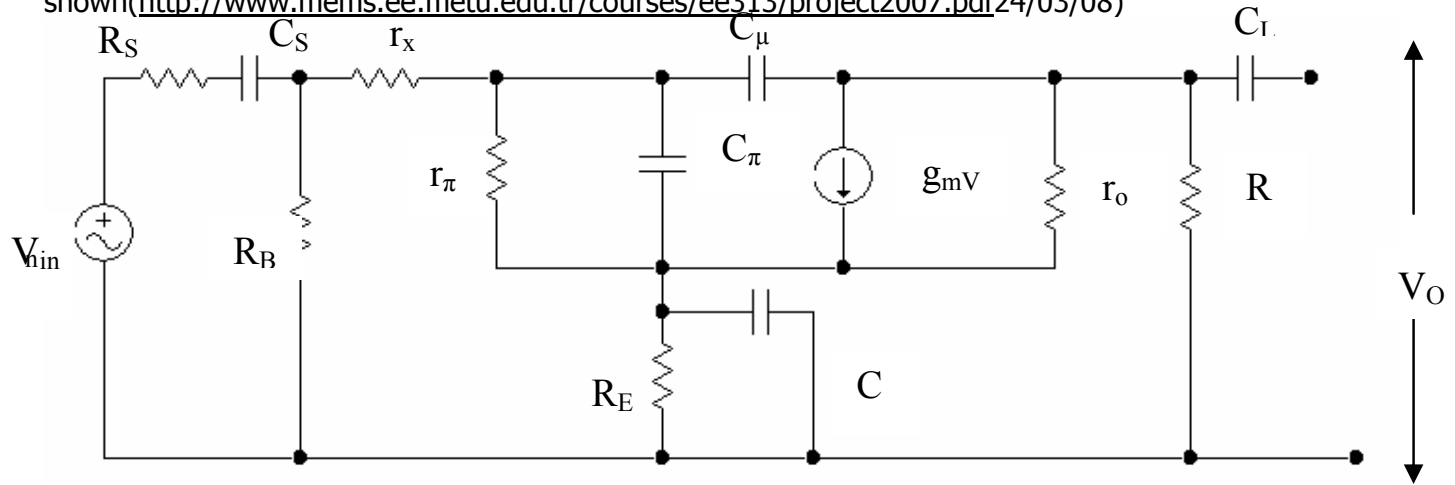

Fig 1.0 Common Emitter Amplifier Hybrid pi -model for low and high

Where $C_{\mu}$ represents the capacitance of the base collector junction. Since the base. Experimentally, $C_{\Pi}$ can be determined from a measurement of $f_{T}$, the frequency at which the common emitter short circuit current gain drops to unity. This is given by 
Bajopas Volume 2 Number 1 June, 2009

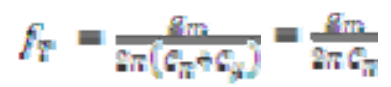

$$
\begin{aligned}
& \text { Where } g_{m}=\frac{V_{T}}{V_{T}} \text { and } V_{T}=\frac{m_{i}}{g_{n}}
\end{aligned}
$$

The voltage gain is given by (Benedict, 1976)

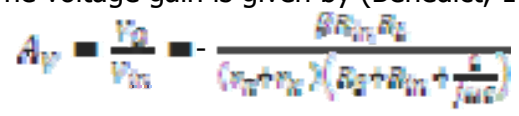

Where $\quad R_{i n}=R_{e} f /\left(x_{i}+x_{n}\right)$

At frequencies so high that $\quad|1 /| j \omega c \mid \notin\left(E_{g}+R_{i n}\right)$, the term $\frac{1}{j \omega \varepsilon}$ can be neglected and the gain approaches

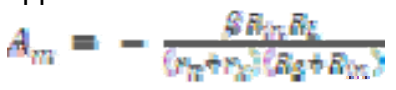

at a limit as $\omega \rightarrow \sim$.Multiplying both the numerator and denominator of equation (2) by $/ \omega c$, we get

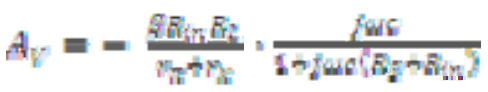

Next we define the parameter $\omega_{1}$ as

$\omega_{1}=\frac{1}{\left\langle B_{y} n_{i n} i_{i n}\right.}$

Using this parameter in equation (5), we have

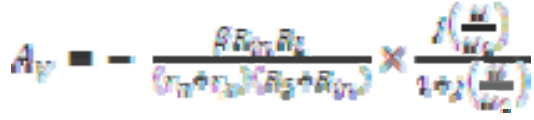

Comparison of equations 7 with 4 shows that equation (7) can be written as

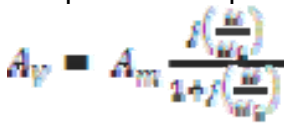

Where $A_{m}$ is the high frequency asymptote defined in equation (4) .Multiplying both the numerator and denominator of equation $(8)$ by $\left(\frac{w}{s}\right)$, we have

$$
A_{Y}=A_{m} \frac{1}{1-i\left(\frac{\pi i n}{i n}\right)}
$$

At high frequencies

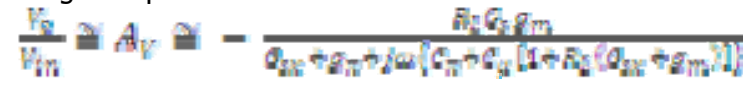

$$
\begin{aligned}
& \text { Let } \quad G_{i}=f_{i}+G_{j i}\left[1+R_{L}\left(G_{i x}+g_{m}\right)\right]
\end{aligned}
$$

The effect of capacitance $C_{p k}$ is increased by the factor in square brackets. This phenomenon is known as miller effect. Equation (10) becomes

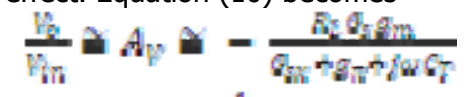

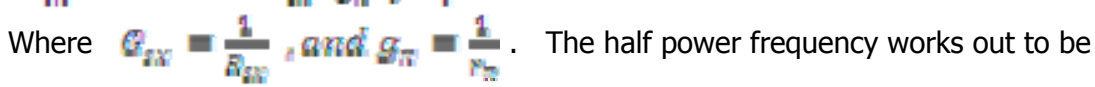

$$
\omega_{z}=\frac{G_{n} h_{i n}}{\sigma_{T}}
$$

The normalized form of equation (12) is given by

$$
\begin{aligned}
& A_{w}=A_{\mathrm{m}} \frac{1}{1-\gamma\left(\frac{\mathrm{r}}{\mathrm{w}}\right)}
\end{aligned}
$$

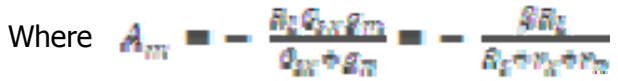

An approximate expression for gain that is valid at all frequencies is given by (Paul, 1982) 
Bajopas Volume 2 Number 1 June, 2009

$A_{p}=A_{m}\left(\frac{1}{\left(1+\frac{w_{w}}{1-\frac{\pi}{w i}}\right)}\right)$

\section{Bandwidth}

The bandwidth of an amplifier is defined as the difference in frequency between the lower and upper frequencies at which the gain is $3.0 \mathrm{~dB}$ down on its maximum value (Edward, 2006 ). The frequency range from $F_{k}$ to $F_{F}$ in the figure below is called the bandwidth of the amplifier stage. Where $F_{L}$ is referred to as the lower $Z_{-} G E$ frequency and $F_{H}$ is the upper $\mathbb{B}_{-} d E$ frequency (Jacob and Halkias, 1991).

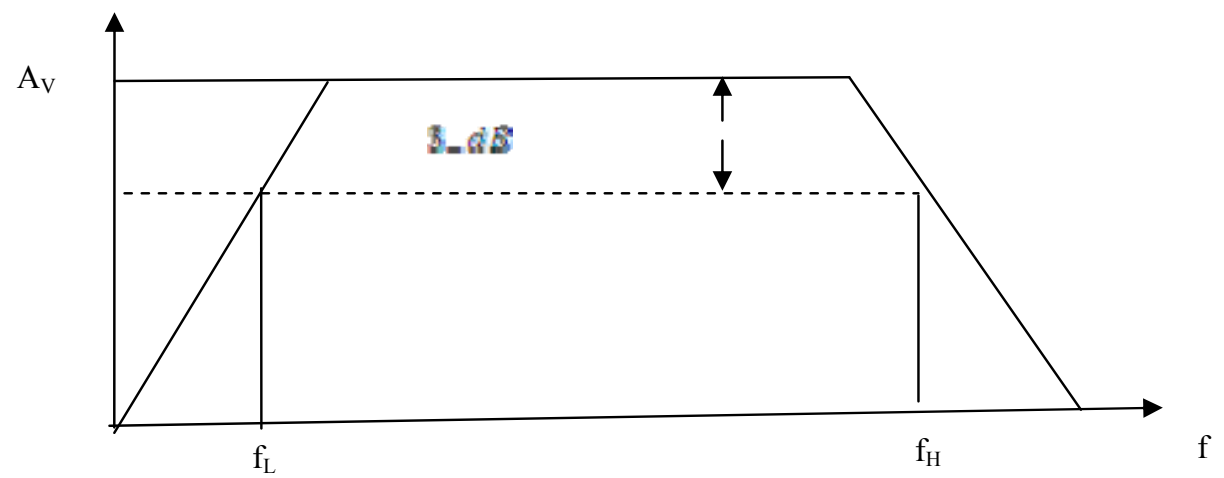

Figure 2.0 A graph of Gain(dB) versus frequency $(\mathrm{Hz})$

The bandwidth of each system is determined by $f_{E}$ ard $f_{N}$, that is ( Robert L Betal,2006). Bandwidth $(B W)=f_{k}-f_{k}$

The gain bandwidth product for an amplifier is defined as the product of the open loop gain (constant for a given amplifier ) and its 3-dB bandwidth. It is given by (htt://en.wikipedia.org/wiki/Gain-bandwidth product, $18 / 04 / 08$ )

$B E I K=A_{m}\left(f_{H}-f_{E}\right)$

Where $A_{w}$ is the mid frequency gain.

\section{Emitter-Coupled Amplifier}

The emitter coupled pair can be regarded as a two-stage circuit. The coupling between the stages is provided by the emitter resistor $\mathbb{R}_{\varepsilon}$ which carries the combined emitter currents of the pair (Benedict R.R, 1976)

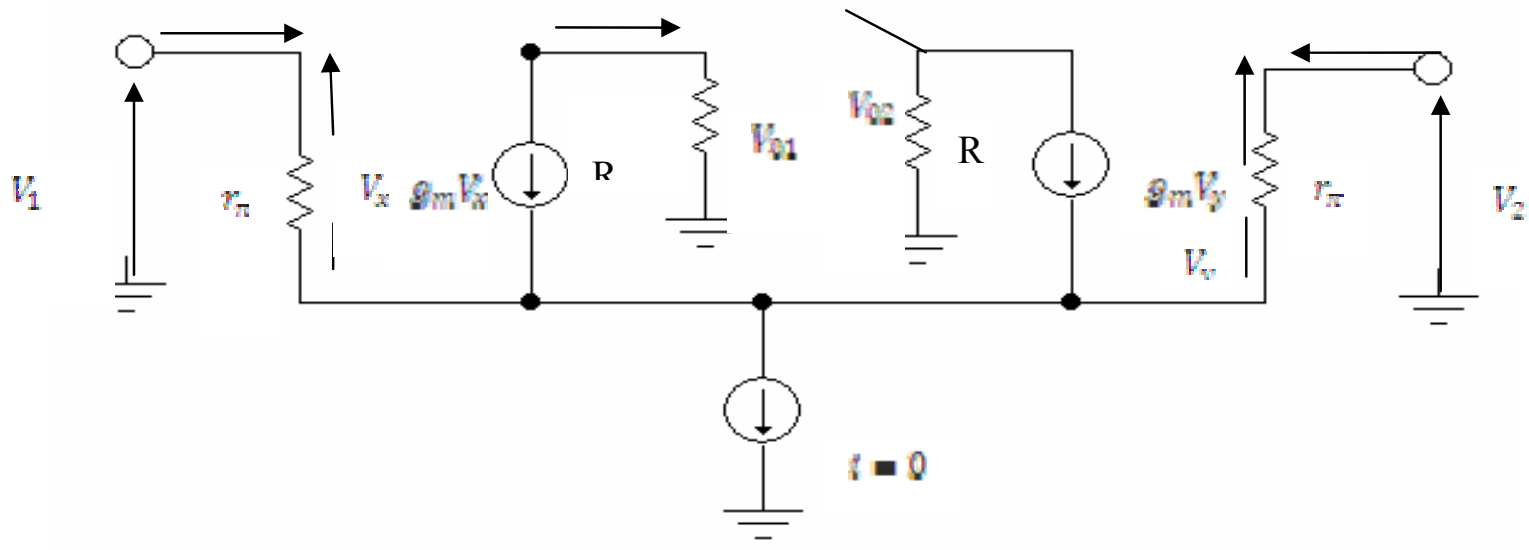

Figure 3.0: Hybrid pi- model for Emitter- Coupled Amplifier

Suppose that we make an incremental analysis of the amplifier above. We are interested in the

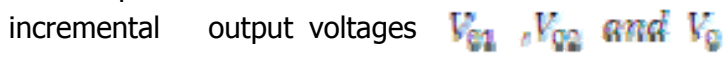
as functions of $\mathrm{I}_{\mathrm{f}}$ ard $\mathrm{V}_{\mathrm{g}}$ (regarded here as incremental values) and the circuit parameters. The results of this analysis, in which we use the simplification $\mathbb{R}_{\mathrm{fP}}=\mathbb{R}_{\mathrm{E}}(\mathbf{1}+\beta)$ are as follows: 
Bajopas Volume 2 Number 1 June, 2009

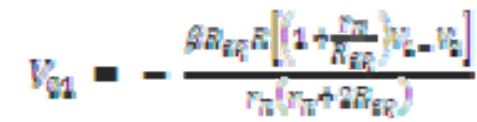

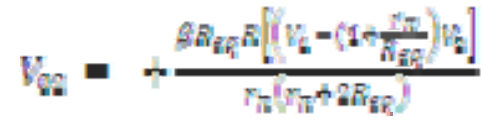

Kirchhoff's voltage law shows that

$$
\begin{aligned}
& b_{\mathrm{Q}}=\mathrm{b}_{\mathrm{C}_{2}}-y_{\mathrm{O}_{3}}
\end{aligned}
$$

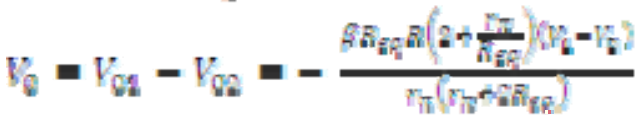

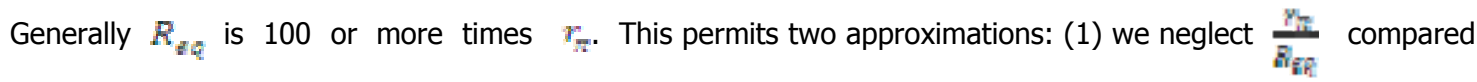
to 1 or 2 in the numerators and (2) we neglect $r_{\pi}^{2}$ compared to $2 \pi R_{\sigma S}$ in the denominators. Using these approximations and the relation $\beta=\eta_{\pi} \xi_{m}$, we obtain from equations 20, 21 and 22 (Benedict, 1976).

$$
\begin{aligned}
& \mathrm{P}_{\mathrm{M}} \cong-\frac{\mathrm{Im}}{\mathrm{g}} R\left(\mathrm{~V}_{1}-\mathrm{B}_{2}\right) \\
& V_{\text {Q }} \propto-\frac{I_{m}}{g} R\left(V_{1}=V_{Q}\right)
\end{aligned}
$$

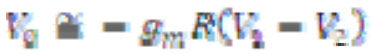

Each output voltage is proportional to the difference $V_{1}-V_{2}$. From the signs we see that the output at $V_{\mathscr{\alpha}}$ is inverted or has the opposite phase form of $Y_{01}$ and has the same magnitude as $Y_{01}$. This feature is used in the phase splitter.

\section{The Distributed Amplifier}

A distributed amplifier is a very resourceful example of distributed circuit design that incorporate transmission line theory into traditional amplifier design in order to arrive at an amplifier with a larger gain bandwidth product that is realizable by conventional circuits

(htt://en.wikipedia.org/wiki/distributed-amplifier 18/04/08) A distributed amplifier consists of two transmission lines and multiple transistors that provide

$$
A_{i}=\frac{n}{2} g_{m} R E
$$

gain through multiple signal paths that amplify the forward travelling wave. Each transistor adds power in phase to the signal at the top point on the output line. Each pathway provides some gain and therefore the whole amplifier is capable of providing a higher gain bandwidth product than a conventional amplifier. For input and output with equal characteristics impedance, the gain of the distributed amplifier can be approximated as (Ali, 2003)

Where: $\quad n=$ Number of transistors, $g_{m}$ Transconductance of each transistor

$\mathbb{R}=$ Characteristics impedence of the input and output lines

$L=$ End to end loss in the in transmission line

There is an optimum number of sections that maximizes the gain, given as (Park, 2003)

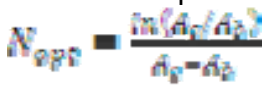

Where $A_{b}$ and $A_{c}$ are the attenuation per section of the transmission lines associated with the base and the collector connections respectively. 
Bajopas Volume 2 Number 1 June, 2009

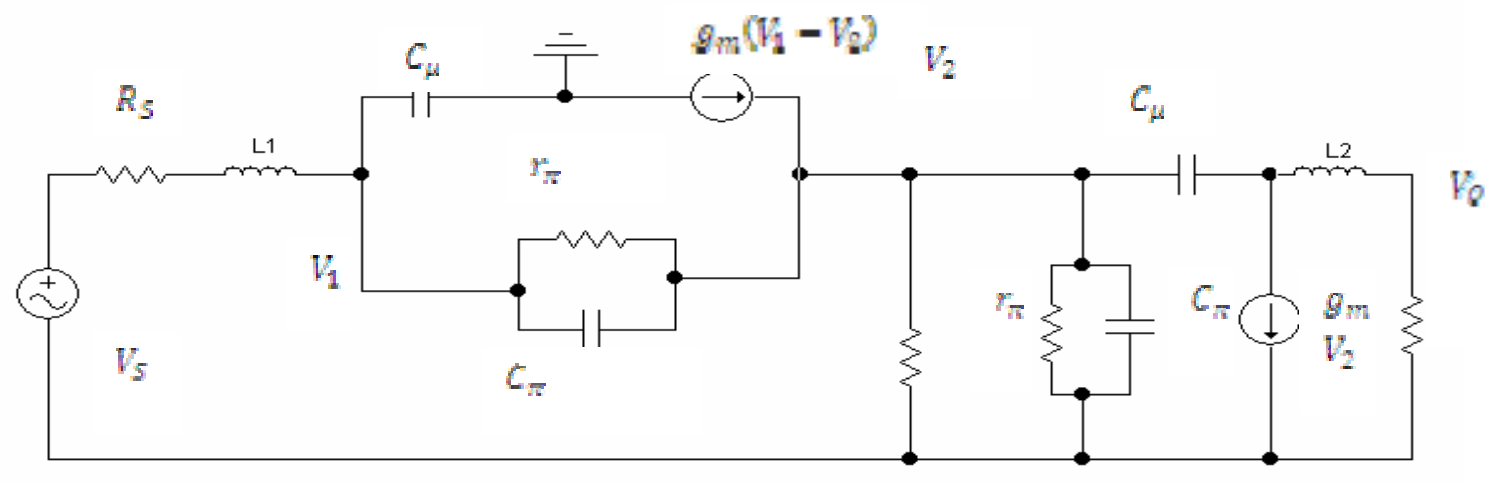

Figure 4.0: Hybrid pi- model of one of the amplifier element of the Distributed Amplifier

\section{MATERIALS AND METHODS}

The method employed here was the Computer Simulation using one of the latest versions (2007) of NI-Circuit Design ( Multisim - Electronics Workbench Software). First the input and output data of the selected transistor (MPS5179) were determined and the characteristics curves were drawn. From the characteristics curves, the

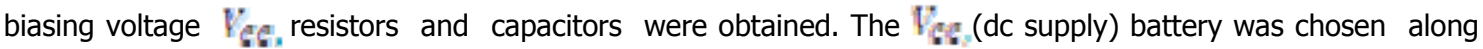
the $V_{\zeta \varsigma}$, axis. The bias voltage $V_{G E}$, was approximated to be $\frac{1}{G} V_{G S}$. By selecting a particular value of $I_{B}$ ,the Q-point was located as the intersection of the selected values. The value of $I_{C}$ at the Q-point was obtained from the curve. Using the values of $I_{G} V_{G G}, V_{G E}$ and $I_{B}$, the biasing resistors were computed. $V_{E}$ is approximately $\frac{1}{10} \mathbb{F}_{G G}$ (Green,1992).

Using Ohm's law

$R_{E}=\frac{V_{E}}{F_{i}}$

And from the output loop (Edward $\mathrm{H}, 2006$ )

$R_{G}=\frac{V_{C C}-Y_{C E}-V_{E}}{i_{G}}$

The collector emitter current amplification factor is given by

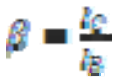

The biasing voltage is given by (Green,1992)

$V_{\text {BE }}=V_{E}+V_{\text {BE }}$

For silicon $V_{E E}=0.7 \mathrm{~V}$

$R_{R_{2}}=\frac{1}{12}\left(Q R_{E}\right)$

Where $R_{E}=\frac{\text { thas }}{10 i c}$

From potential divider (Edward, 2006)

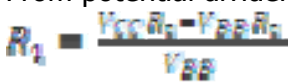

The coupling capacitor $C$ and the emitter bypass capacitor $C_{E}$ were obtained from

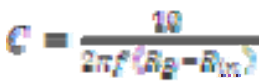

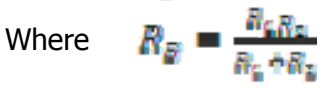

$R_{\text {in }}=R_{1} / f R_{2} / f R r_{r}$

$r_{\pi}=\beta r_{r}$

$C_{E}=\frac{\operatorname{sil}}{\sin \theta_{E}}$ 
The inductance $L$, the shunt capacitance $C$ per unit length of the transmission lines and the characteristics impedance of the line $Z_{0}$ are related by the expression (Delaney $C F, 1980$ )

$z_{0}=\sqrt{\frac{5}{6}}$

The delay time per unit length for the T-line is given by:

$T_{d}=\sqrt{E_{\mathrm{r}}}$

Using the above equations, the values of the parameters were obtained as follows

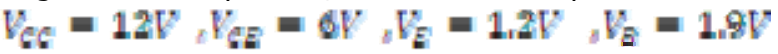

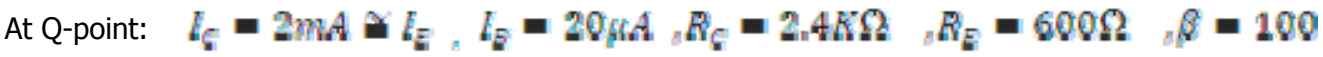

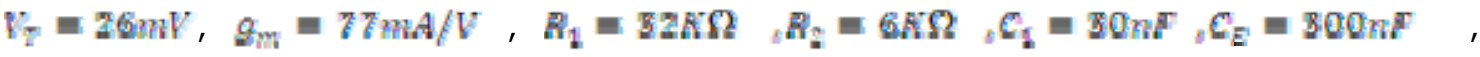

$E=25 H W, R=50 \Omega$ and $\mathrm{C}=10 \mathrm{mF}$

RESULTS

The data obtained from the amplifiers designed using the above parameters were plotted using the ORIGIN 50 and the curves obtained are shown below:

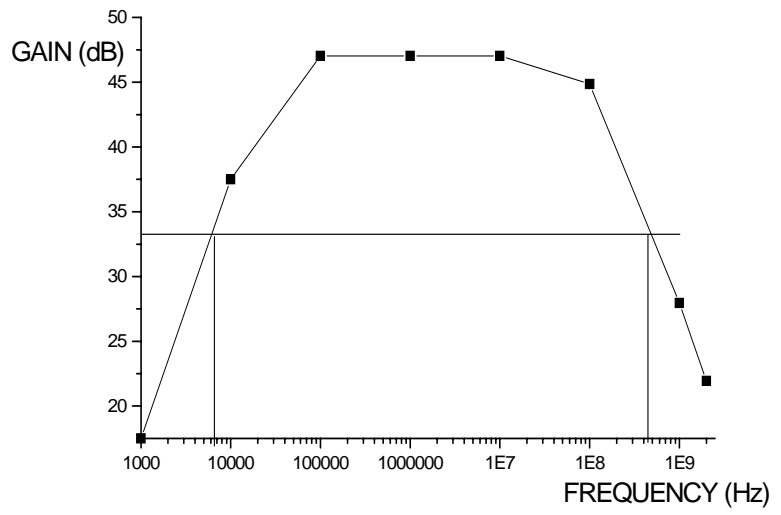

Figure 5.0: A Graph of Gain (dB) versus Frequency $(\mathrm{Hz})$ for Single Stage Common Emitter Amplifier

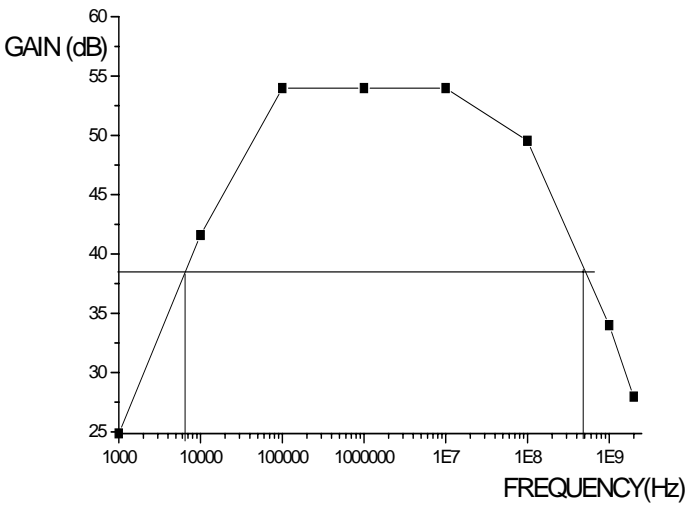

Fig 6.0 A Graph of Gain (dB) versus Frequency (Hz) for Emitter- Coupled Amplifier 
Bajopas Volume 2 Number 1 June, 2009

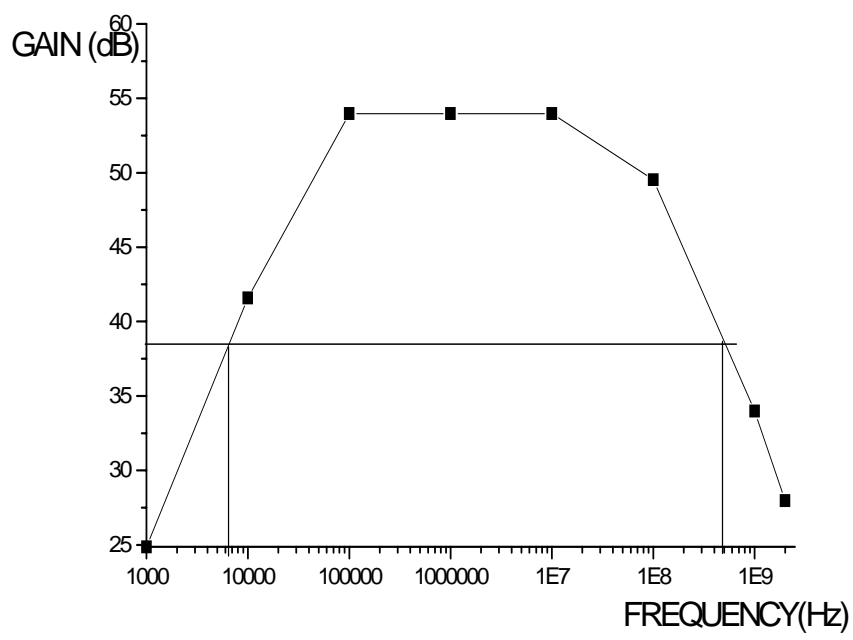

Fig 7.0.A graph of Gain (dB) Versus Frequency (Hz) For Distributed Amplifier

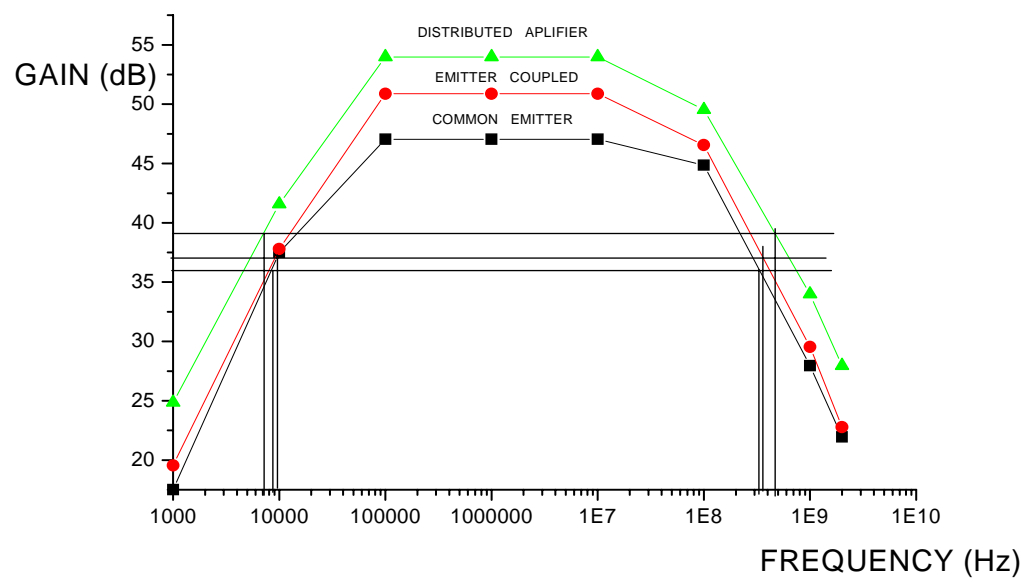

Figure 8.0. Comparison Graphs of Gain (dB) versus Frequency (Hz) for Common Emitter Amplifier, Emitter-coupled Amplifier and Distributed Amplifier.

Table 1.0 Summary of Measurement Result from the Graphs

\begin{tabular}{|c|c|c|c|c|c|}
\hline $\begin{array}{l}\text { 㞻 } \\
\text { 苛 } \\
\frac{\mathbf{a}}{\Sigma}\end{array}$ & 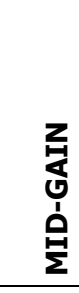 & $\underset{4}{\stackrel{\mathbf{N}}{\mathbf{Z}}}$ & $\underset{4}{\stackrel{\mathbf{N}}{\mathbf{Z}}}$ & 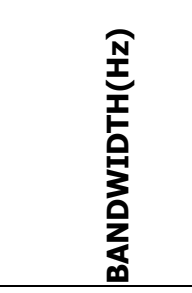 & 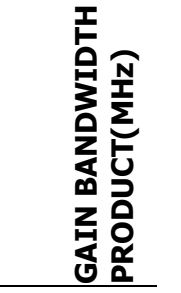 \\
\hline SINGLE STAGE COMMON & & 8045.71383 & 433189078.7 & 433181033 & 97465.73243 \\
\hline AMPLIFIER & 225 & & & & \\
\hline EMITTER COUPLED & 350 & 7081.44444 & 443229601.5 & 443222520.1 & 155127.882 \\
\hline \multicolumn{6}{|l|}{ AMPLIFIER } \\
\hline DISTRIBUTED AMPLIFIER & 500 & 6853.39507 & 454978238.4 & 454971385.0 & 227485.693 \\
\hline
\end{tabular}




\section{SUMMARY AND CONCLUSION}

The equations (28) to (41) were used to design the amplifiers. A study of frequency response of each amplifier was carried out and the results obtained were tabulated. The graphs of such tabulations were plotted as shown above. The distributed amplifier was modified in such a way that it has a relaxed gain bandthwidth trade- off compared to the conventional amplifier since the parasitic capacitances of the

\section{REFERENCES}

Ali, M.H (2003)' Design of an active antenna array Based on the Distributed Amplifier'Ph.D Thesis, Bayero University Kano

Bachman, A and Hajimiri, A (2004),' Bandwidth Enhancement Technique for Transimpedence Amplifier' IEEE-Magazine.,2004.

Behzad, R (2003),' Design of Integrated circuits for Optical Communication' McGraw-Hill Higher Education New York 1-2pp.

Benedict, R.R (1976),' Electronics for Scientists and Engineers', Prentice-Hall International 171-223pp.

Charles Q. and Emilio A. (2003), $40 \mathrm{GHz}$ Transimpedence Amplifier with Differential outputs using InP-InGaAs Heterojunction Bipolar Junction Transistor', IEEE Journal of Solid State Circuits Vol.38 NO.9 September 2003.

Delaney C.F (1980), 'Electronics for the Physicist' Ellis Horwood Limitd, Chichester England 192194pp transistors are absorbed into the transmission lines or the LC ladder filter to become part of the passive network. Results from table 1.0 shows that there is a significant improvement in the gain, bandthwidth, and gain- bandthwidth product of the distributed amplifier. Distributed amplifiers are used in many RF and high data rate communication systems including satellite transceivers, pulsed radar systems, optical receivers and so on.

Edward H. (2006),'Electrical and Electronic Technology', Dorling Kingsley (India) pvt.Itd $362 \mathrm{p}$

Green D.C (1992),'Electronics 1V' Longman Group UK Limited 45-50pp

htt://en.wikipedia.org/wiki/Gain-bandwidth product, 18/04/0814

http://www.mems.ee.metu.edu.tr/courses/ee313/proj ect2007.pdf 24/03/08

htt://en.wikipedia.org/wiki/distributed-amplifier 18/04/08

Jacob M. and Halkias C. (1991), 'Integrated Electronics ' McGraw-Hill Publishing Company Limited, New Delhi.378p

Paul, M.C (1982),' Analysis and Design of Integrated Electronic circuits' Harper and Row, Publishers, London, 526p

Robert, L.B. (2006),' Electronic Devices and Circuit Theory' ,Dorling Kindersley (India) Pvt.Itd $499 p, 216 p$

Park, J. (2003) "Design of an RF CMOS Ultrawideband Amplifier Using Parasitic Aware Synthesis and Optiimization".PhD Thesis, University of Washington 17p. 